\title{
Medical Occupation
}

National Cancer Institute

\section{Source}

National Cancer Institute. Medical Occupation. NCI Thesaurus. Code C19254.

Occupations within the field of medicine. 